\title{
AGE-RELATED DIFFERENCES IN CO-MORBIDITY NUMBER, FUNDUS ATHEROSCLEROSIS LEVEL AND THE SERUM VALUES OF GSH-PX, HS-CRP AND HDL-C IN ELDERLY CHINESE PATIENTS
}

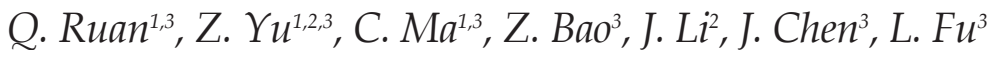

\begin{abstract}
Background: The ApoE genotype, atherosclerosis, status of inflammation, oxidative stress and co-morbidity may be detrimental to the elderly. Objectives: To identify biomarkers of aging. Setting: All subjects were Chinese elderly in Shanghai. Subjects: 549 outpatients ( 489 male, 60 female), divided into $\leq 74$ year-old, 75-84 year-old and the oldest old ( $\geq 85$ year-old ) groups. Methods: A univariate analysis was used to investigate 5 age-related categorical variables and 26 continuous variables. The related variables were used to find the independent biomarkers of aging by Multivariate logistic regression analyses. Results: The serum values of Glutathione peroxidase, HDL-C and $\mathrm{C}$ reactive protein, the number of co-morbidities and fundus atherosclerosis level were the main independent age-associated factors that influenced aging. Compared with $\geq 85$ year-old individuals, $\leq 74$ year-old individuals had fewer co-morbidities [OR, $0.757(95 \% \mathrm{CI}, 0.636,0.902)]$, lower grades of fundus atherosclerosis [Grade 0: OR, 26.059 (95\% CI, 4.705, 144.324)] and [Grade I: OR, 8.539 (95\% CI, 3.555, 20.513)] and lower serum levels of HDL-C [OR, 0.127 (95\% CI, 0.037, 0.433)]. However, 75-84 year-old patients had significantly lower plasma levels of GSH-px [OR, 0.986, (95\% CI, 0.972, 1.00)], HDL-C [OR, 0.158 (95\% CI, 0.054, 0.457)] and HsCRP [Grade I: OR, 8.516 (95\% CI,1.630, 44.484)], [Grade II: OR,7.699 (95\% CI,1.544, 38.388)] and [Grade III: OR,7.251 (95\% CI,1.346, 39.070)]. Conclusion: The oldest old patients had significantly high antioxidant capability and serum HDL-C level. However, these patients also had a significantly high systemic inflammation, number of co-morbidities and grades of fundus atherosclerosis.
\end{abstract}

Key words: Lipoprotein and apolipoprotein, antioxidant enzymes, inflammatory cytokine, fundus atherosclerosis, co-morbidity.

\section{Introduction}

Aging is characterized by a time-dependent progressive decline in organismal physiological reserves and by an increased vulnerability to age-associated diseases, which can lead to death. A number of peripheral blood proteins may involve in aging process and be potential biomarkers of aging. Centenarians were shown to have a significantly low oxidative stress grade compared with a control group of 70-80 yearold elderly persons (1). An age-related increase in enzymatic antioxidant activities was seen in subjects from less than 60 years of age to Italian nonagenarians (2). Elevated inflammatory markers, especially IL-6, have been shown to be closely associated with multiple age-related diseases and all-cause mortality (3). A high

1. Department of Aging, Antiaging and Cognitive Function, Shanghai Institute of Geriatrics and Gerontology, Shanghai 200040, China; 2. Department of Geriatrics, Huadong Hospital, Shanghai Medical College, Fudan University, Shanghai 200040, China; 3. Research Center of Aging and Medicine, Department of Aging and Shanghai ey Laboratory of Clinical Geriatrics, Huadong Hospital, Shanghai Medical College, Fudan University, Shanghai 200040, China.

Corresponding Author: Zhuowei Yu, Huadong Hospital, Shanghai Medical College, Fudan University, 221 West Yan An Road, Shanghai 200040, P.R. China. Tel: 86-2162483180; Fax: 86-21-62484981; E-mail: hdyuzhuowei@163.com level of IL-6 long-term was associated with older age, obesity, smoking, lower physical activity and lower high-density lipoprotein cholesterol (HDL-C) (4). In aging men, systemic inflammation was associated with normal aging and age-related diseases, such as coronary atherosclerosis (AS) and lower urinary tract symptoms $(5,6)$. High plasma high sensitivity $C$ reactive protein (HsCRP) concentrations, which are associated with sex, age, waist circumference and systolic blood pressure, were similar between Inuit persons and Caucasians, despite their differing lifestyles (7).

A high serum level of HDL-C is a characteristic feature of centenarians and is associated with better survival in frail, community-living elderly $(8,9)$. In centenarians, a progressive decline in plasma HDL was shown to be associated with cognitive dysfunction and elevated CRP and IL-6 levels $(8,10)$. ApoE polymorphisms are important determinants of blood lipids. ApoE $\varepsilon 4$ allele carriers have the highest total cholesterol $(\mathrm{TCH})$ and low-density lipoprotein cholesterol (LDL-C) levels and are susceptible to cardio-cerebrovascular disease, dementia and Alzheimer's disease. Multiple studies have shown that long-lived populations have lower 
$\varepsilon 4$ frequencies when compared with a general or middle-aged population (11). The mechanisms of ApoEregulated aging and age-related diseases are related to the antioxidant and anti-inflammatory properties of ApoE (12). Apolipoprotein C-III (ApoC-III) is the major component of triglyceride (TG)-rich lipoproteins. Lower serum levels of ApoC-III had a protective effect against cardiovascular disease and metabolic syndrome (13). ApoB protein level, which is mediated by the ApoE polymorphism, may be a useful predictive factor for cardio-cerebrovascular complications $(10,14)$. An increase in serum ApoA-I prevented AS and enhanced longevity through antioxidative and anti-inflammatory effects (15). The up regulation of ApoJ increases stress resistance and extends lifespan in Drosophila, lower in a sample of human centenarians $(16,17)$. Although a number of study showed many peripheral blood proteins, including inflammatory cytokines, Lipoprotein and apolipoprotein, Co-morbidity, oxidative stress and Fundus atherosclerosis grade may be potential predictors of aging. However, the study about these potential biomarkers dynamic changes in subjects with different age is little. The current study was to investigate ageassociated changes of these potential aging biomarkers

\section{Methods}

\section{Sample}

The study was conducted at Huadong Hospital. Between June 1, 2012, and July 31, 2012, a total of 549 outpatients (48-103 years old) received physical examinations with complete medical histories. All the subjects had given written informed consent to participate in the study. TCH, TG, HDL-C and LDL-C levels were determined using the standard enzymatic colorimetric technique. BMI was used to indicate weight change. Subjects in the experiment had more than 12 years of education and similar life styles (a typical shanghainese diet, nearly no cigarette smoking or alcohol consumption). The categorical variant "age" was divided into three stratifications, $\leq 74(66.89 \pm 0.49)$, 75-to 84-yearold $(80.12 \pm 0.22)$ and $\geq 85$ years $(89.92 \pm 0.24)$, and the degree of sclerotic change in fundus AS was graded as 0 , I and II based on fundus photographs according to our previous study (18). Metabolic syndrome was described as "yes" or "no" according to the definition of the Joint Interim Statement (19). The Medical Ethics Committee of Huadong Hospital of Shanghai Medical College, Fudan University approved this study.

\section{Study parameters}

\section{Co-morbidity profile}

Participants in this study were asked whether they had a physician's diagnosis of the following 18 diseases that are classified as chronic based on the International Classification of Diseases (ICD): hypertension, dyslipidemia, obesity, diabetes, coronary heart disease, other heart diseases, venous insufficiency, stroke, epilepsy, hypothyroidism, hyperthyroidism, chronic renal disease, anemia, chronic pulmonary obstructive disease, liver disease, arthrosis, prostatic disease and cancer. Each recorded disease was added to generate a score ranging from 0 to 18 .

\section{Serum Cytokines}

Serum from a 2-mL fasting peripheral blood sample was collected at $2000 \times \mathrm{g}$ for 10 minutes in the morning and rapidly stored at $-80^{\circ} \mathrm{C}$. The serum concentrations of cytokines were determined using Bio-Plex Human 6-Plex (IL-1 $\beta$, IL-6, IL-4, IL-10, TNF- $\alpha$ and RANTES) kits and Bio-Plex Human 1-Plex (TGF- $\beta$ ) kits (Laboratories, Hercules, California, USA). To pre-wet the wells of a 96-well filter plate, coupled beads, serum samples, antibodies and streptavidin-PE (each $50 \mu \mathrm{L}$ ) were prepared and added to the wells one after another after two washes, according to the manufacturer's instructions. The samples were run in duplicate using a Bio-Plex MAGPIXTM (Bio-Rad Laboratories, Inc., US). The plate was read using Bio-Plex Manager software version 6.0. Objective concentration $(\mathrm{pg} / \mathrm{mL}$ ) was too low to measure, so fluorescence intensity was used as the relative concentration.

\section{Lipid peroxidation and activity of antioxidant enzymes}

The lipid peroxide (LPO) concentration in the serum was determined quantitatively by using an LPO Assay Kit (Nanjing Jianchen Bio, Jiang su, China) according to the protocol of the manufacturer. The product absorbance of a molecule of LPO and two molecules of chromogenic agent was read at a wavelength of $450 \mathrm{~nm}$ with a using TECAN Sunrise ${ }^{\mathrm{TM}}$ immediately after incubation at $45^{\circ} \mathrm{C}$ for $60 \mathrm{~min}$. The concentration of LPO $(\mu \mathrm{mol} / \mathrm{L})$ was calculated according to a formula.

Superoxide dismutase (SOD) activity was determined using an SOD Water-Soluble Tetrazolium Salt Assay Kit (Nanjing Jianchen Bio, Jiangsu, China) according to the manufacturer's instructions. The corresponding SOD activity at 50\% inhibition in the response system of 20 $\mu \mathrm{L}$ of SOD solution, $20 \mu \mathrm{L}$ of serum samples and 200 $\mu \mathrm{L}$ of substrate was defined as one activity unit (U). The change in SOD activity was determined by measuring the absorbance at $450 \mathrm{~nm}$ using using TECAN Sunrise ${ }^{\mathrm{TM}}$.

Peroxidase (POD) activity was measured using a POD Assay Kit (Nanjing Jianchen Bio, Jiangsu, China). The corresponding POD activity of $1 \mu \mathrm{g}$ of substrate $\left(\mathrm{H}_{2} \mathrm{O}_{2}\right)$ in $1 \mathrm{~mL}$ of serum at $37^{\circ} \mathrm{C}$ for $1 \mathrm{~min}$ was defined as one activity unit (U). The change in POD activity was 
determined by measuring the absorbance at $420 \mathrm{~nm}$ using using TECAN Sunrise ${ }^{\mathrm{TM}}$.

Catalase (CAT) activity was measured using a CAT Assay Kit (Nanjing Jianchen Bio, Jiangsu, China). Dissociation of $1 \mu \mathrm{mol}$ of substrate $(\mathrm{H} 2 \mathrm{O} 2)$ in $1 \mathrm{~mL}$ of plasma at $370 \mathrm{C}$ for $1 \mathrm{~min}$ was defined as one activity unit (U). The chromogenic agent ammonium molybdate combined with surplus $\mathrm{H} 2 \mathrm{O} 2$ substrate in the response system produced a yellow-colored product that was measured at $405 \mathrm{~nm}$ using a Hitachi F-2000 fluorescence spectrophotometer.

Glutathione peroxidase (GSH-px) activity was measured using a GSH-px Assay Kit (Nanjing Jianchen Bio, Jiangsu, China), according to the manufacturer's protocol. When $4 \mu \mathrm{L}$ of whole blood reacted with the substrate (H2O2) at $370 \mathrm{C}$ for $5 \mathrm{~min}$, a GSH concentration decline of $1 \mu \mathrm{mol} / \mathrm{L}$ in the response system was defined as one activity unit (U), after deducting the effect of the non-enzymatic response. GSH combined with the chromogenic agent dithiobisnitrobenzoic acid (DTNB) produced stable yellow five-glucosinolate two-nitro benzoic acid anions that were measured at $412 \mathrm{~nm}$ using a Hitachi F-2000 fluorescence spectrophotometer.

\section{Serum apolipoproteins}

The levels of different apolipoproteins were detected using an ELISA KIT (ApoE, ApoH; abcam, Cambridge, UK), an ApoJ kit (R\&D) and a Milliplex 5-plex kit (ApoA I, ApoA II, ApoB, ApoC II, ApoC III) (Millipore, MA, US). For the ELISA test, serum samples were diluted into diluent at 1:400. Apolipoprotein standards or human apolipoprotein samples $(50 \mu \mathrm{L})$ were added to each well of a microplate, and the assay was run according to the manufacturer's protocol. After finishing the experiments, the absorbance was immediately read on a microplate reader at a wavelength of $450 \mathrm{~nm}$. The mean values of the duplicate or triplicate readings for each standard and sample were calculated, and a standard curve using eight standard concentrations on the $\mathrm{x}$-axis and the corresponding mean $450 \mathrm{~nm}$ absorbance on the $\mathrm{y}$-axis was generated. The best-fit line was determined by regression analysis using log-log or a four-parameter logistic curve-fit. The unknown sample concentration from the standard curve was multiplied by the dilution factor. The average intra- and inter-assay CVs were $4.6 \%$ and $7.4 \%$, respectively. The minimum detectable dose of apolipoprotein was typically $\sim 0.03 \mu \mathrm{g} / \mathrm{mL}$, and the standard added value was $0.05-0.5 \mu \mathrm{g} / \mathrm{mL}$. For the Milliplex 5-plex apolipoprotein assay, similar as for the Bio-Plex Human 6-Plex assay with Bio-Plex MAGPIXTM, the coupled beads, serum samples $(5 \mu \mathrm{l})$ and antibodies were prepared according to the manufacturer's instructions. The samples were run in duplicate using the Luminex 200TM System (Luminex, Austin, US). The objective concentration unit was $\mathrm{ng} / \mathrm{mL}$.

\section{Serum HsCRP}

The serum HsCRP concentrations of the study subjects were determined with an Hs-CRP kit (Jun Shi Bioscientific, Shanghai, China) using an immunonephelometric assay that had been improved to provide greater sensitivity; this has been previously described in detail (20). The World Health Organization CRP reference standard was used. The intraassay and interassay coefficient of variation for this assay were $\leq 4.0 \%$ and $\leq 5.0 \%$, respectively. The technicians were blinded to the case-control status of the samples. The normal value of Hs-CRP was $<0.3 \mathrm{mg} / \mathrm{L}$. The study subjects with Hs-CRP $\geq 10$ were considered to be suffering from an acute infection and were excluded from the study.

\section{ApoE genotyping}

Genomic DNA was extracted from peripheral whole blood samples using standard methods. Two singlenucleotide polymorphisms (SNPs; rs429358 and rs7412) were genotyped to identify APOE genotypes composed of the APOE $\varepsilon 2, \varepsilon 3$ and $\varepsilon 4$ alleles using a SNaPshot minisequencing assay (21). As $\varepsilon 2$ is regarded as a protective factor and $\varepsilon 4$ is regarded as a risk factor ( $\varepsilon 3$ is regarded as neutral), persons with the $\varepsilon 2 / 4$ genotype were removed from the sample as their inclusion might have weakened the contrast between $\varepsilon 2$ and $\varepsilon 4$, which was the primary interest of the study. For this investigation, the APOE group composition was as follows: $\varepsilon 2=\varepsilon 2 / 2+\varepsilon 2 / 3 ; \varepsilon 3=$ $\varepsilon 3 / 3$; and $\varepsilon 4=\varepsilon 3 / 4+\varepsilon 4 / 4$.

\section{Statistical Analysis}

To investigate the association between single categorical variables, such as sex, ApoE genotype, metabolic syndrome, the grade of fundus atherosclerosis and Hs-CRP, with age, a chi-squared test was used. Hs-CRP levels were stratified into $<0.3,0.3 \sim 1,1-3$ and $\geq 3$. To investigate the association between single continuous variables, such as BMI, LPO or anti-oxidant enzymes, inflammatory cytokines, lipoproteins, apolipoproteins and number of co-morbidities, with age, an ANOVA (for Gaussian distributions) or Kruskal-Wallis test (for non-Gaussian distributions) was used. The most commonly missing values were those for variable SOD and accounted for $12.02 \%$ of the subjects. All significant associations of categorical and continuous variables with age were further analyzed using multivariate logistic regression analyses; these studied the same effects after adjusting for multiple covariates. A p value $<0.05$ was considered statistically significant. All analyses were performed using SPSS 18.0 software. 
Table 1

The associations between single categorical variables and age by univariate analysis ( $\mathrm{n}=$ sample number)

\begin{tabular}{|c|c|c|c|c|c|c|}
\hline & & All $n=549$ & $\begin{array}{c}\leq 74 \text { years old } \mathbf{n}= \\
176(32.06 \%)\end{array}$ & $\begin{array}{c}\text { 75- to 84-year- } \\
\text { old } n=203 \\
(36.98 \%)\end{array}$ & $\begin{array}{l}\geq 85 \text { years old } n= \\
\quad 170(30.97 \%)\end{array}$ & p-value ${ }^{a}$ \\
\hline \multirow[t]{2}{*}{ Sex } & Male & 489 & $162(33.1 \%)$ & $182(37.2 \%)$ & $145(29.7 \%)$ & 0.125 \\
\hline & Female & 60 & $14(23.3 \%)$ & $21(35.0 \%)$ & $25(41.7 \%)$ & \\
\hline \multirow[t]{3}{*}{ ApoE genotype } & $\varepsilon 2$ & 69 & $19(27.5 \%)$ & $33(47.8 \%)$ & $17(24.6 \%)$ & 0.287 \\
\hline & $\varepsilon 3$ & 380 & $124(32.6 \%)$ & $138(36.3 \%)$ & $118(31.1 \%)$ & \\
\hline & $\varepsilon 4$ & 71 & $28(39.4 \%)$ & $25(35.2 \%)$ & $18(25.4 \%)$ & \\
\hline \multirow[t]{3}{*}{ AS level } & 0 & 30 & $22(73.3 \%)$ & $5(16.7 \%)$ & $3(10.0 \%)$ & $<0.001$ \\
\hline & $\mathrm{I}$ & 365 & $140(38.4 \%)$ & $135(37.0 \%)$ & $90(24.7 \%)$ & \\
\hline & II & 150 & $12(8.0 \%)$ & $62(41.3 \%)$ & $76(50.7 \%)$ & \\
\hline \multirow[t]{2}{*}{ Metabolic syndrome } & No & 365 & $125(34.2 \%)$ & $118(32.3 \%)$ & $122(33.4 \%)$ & 0.004 \\
\hline & yes & 179 & $49(27.4 \%)$ & $84(46.9 \%)$ & $46(25.7 \%)$ & \\
\hline \multirow[t]{4}{*}{ Hs-CRP } & $<0.3$ & 157 & $59(37.6 \%)$ & $56(35.7 \%)$ & $42(26.8 \%)$ & 0.043 \\
\hline & $0.3 \sim 1$ & 217 & $66(30.4 \%)$ & $90(41.5 \%)$ & $61(28.1 \%)$ & \\
\hline & $1 \sim 3$ & 76 & $19(25.0 \%)$ & $33(43.4 \%)$ & $24(31.6 \%)$ & \\
\hline & $3 \sim 10$ & 22 & $7(31.8 \%)$ & $3(13.6 \%)$ & $11(54.5 \%)$ & \\
\hline
\end{tabular}

a. Pearson chi-squared test

\section{Results}

Table 1 shows the demographic characteristics and percentages of three age stratifications. A univariate analysis of five categorical variables showed that metabolic syndrome $(\mathrm{p}=0.004)$, the grade of fundus AS $(p<0.001)$ and the stratification of HsCRP $(p=0.043)$ had significant associations with age (Table 1). The 75- to 84-year-old group had a higher percentage of metabolic syndrome. The older individuals had higher grades of fundus AS and higher values of HsCRP. However, sex and ApoE genotype were not significantly associated with age (Table 1 ).

The continuous variables analyzed included five redox homeostasis, seven inflammatory, four lipoprotein and eight apolipoprotein parameters as well as the number of co-morbidities and BMI (Table 2). For these continuous variables, a univariate analysis showed that levels of the anti-oxidant enzymes POD $(\mathrm{p}=0.004)$, SOD $(\mathrm{p}=$ $0.003)$ and GSH-px $(\mathrm{p}=0.018)$ and the levels of the proinflammatory cytokine IL-6 $(\mathrm{p}<0.001)$, apolipoprotein AII $(\mathrm{p}<0.001)$, B $(\mathrm{p}=0.039)$, CII $(\mathrm{p}<0.001)$, CIII $(\mathrm{p}=$ $0.002)$ and $H(p=0.008)$, lipoprotein HDL-C $(p<0.001)$ and LDL-C $(\mathrm{p}=0.004)$ and the number of co-morbidities $(p<0.001)$ were significantly associated with age (Table 2). Higher activities of anti-oxidant enzymes, including POD, SOD, lower activity of GSH-px, higher levels of the inflammatory cytokine IL-6, lower levels of ApoAII, B, $\mathrm{CII}$ and CIII, higher levels of ApoH, HDL-C and LDL-C and a greater number of co-morbidities were found in the older patients (Table 2). Other continuous variables, including the levels of LPO, CAT, IL-1beta, IL-4, IL-10, TNFalpha, TGFbeta, RENTS, ApoAI, ApoE, ApoJ, TCH, TG and BMI were not associated with age.

Fifteen variables significantly associated with age were further analyzed by multivariate logistic regression analyses (forward stepwise) to determine the main factors associated with age. The results indicated that grade of fundus atherosclerosis, number of co-morbidities, serum levels of HDL-C and GSH-px and grade of HsCRP were significantly associated with age (Table 3). Compared with individuals $\geq 85$ years of age, individuals $\leq 74$ years of age had significantly fewer co-morbidities [OR, 0.757 (95\% CI, 0.636, 0.902)], lower grades of fundus AS [Grade 0: OR, 26.059 (95\% CI, 4.705, 144.324)] and [Grade I: OR, $8.539(95 \% \mathrm{CI}, 3.555,20.513)]$ and lower serum levels of HDL-C [OR, 0.127 (95\% CI, 0.037, 0.433)] (Table 3). However, there were no significant differences in serum HDL-C levels and HsCRP grades between the two age groups. Compared with individuals $\geq 85$ years of age, 75- to 84-year-old individuals had similar numbers of co-morbidities and similar grades of fundus AS, but 75- to 84-year-old patients had significantly lower serum levels of GSH-px [OR, 0.986, (95\% CI, 0.972, 1.00)], HDL-C [OR, 0.158 (95\% CI, 0.054, 0.457)] and HsCRP [Grade I: OR, 8.516 (95\% CI,1.630, 44.484)], [Grade II: OR,7.699 (95\% CI,1.544, 38.388)] and [Grade III: OR,7.251 (95\% CI,1.346, 39.070)] (Table 3). 
Table 2

The associations between single continuous variables and age by univariate analysis $(\mathrm{n}=$ sample number)

\begin{tabular}{|c|c|c|c|c|c|c|c|c|}
\hline & All & & $\leq 74$ years old & & 5- to 84-year-old & & $\geq 85$ years old & p-value \\
\hline & $\mathbf{n}$ & $\mathbf{n}$ & Median $^{b}$ & $\mathbf{n}$ & Medianb & $\mathbf{n}$ & Median $^{b}$ & \\
\hline LPO & 514 & 163 & $0.701(0.449,0.977)$ & 190 & $0.667(0.475,0.928)$ & 161 & $0.712(0.468,1.088)$ & 0.565 \\
\hline POD & 521 & 166 & $7.716(5.525,11.921)$ & 194 & $7.716(5.648,11.289)$ & 161 & $9.074(6.358,15.787)$ & 0.004 \\
\hline SOD & 483 & 154 & $10.071(8.787,11.342)$ & 186 & $10.573(8.780,11.986)$ & 143 & $10.785(9.600,12.156)$ & 0.003 \\
\hline CATa & 505 & 163 & $52.119 \pm 0.980$ & 193 & $51.731 \pm 0.774$ & 149 & $52.066 \pm 1.084$ & 0.947 \\
\hline GSH-px & 494 & 158 & $72.922(62.277,80.433)$ & 190 & $68.648(42.239,77.294)$ & 146 & $69.623(55.601,81.484)$ & 0.018 \\
\hline IL-1beta & 519 & 166 & $9.000(8.000,10.000)$ & 192 & $9.000(8.000,10.000)$ & 161 & $9.000(8.000,10.000)$ & 0.723 \\
\hline IL-4 & 518 & 166 & $9.000(8.000,9.000)$ & 192 & $8.000(8.000,9.000)$ & 160 & $8.000(8.000,9.000)$ & 0.063 \\
\hline IL-6 & 520 & 166 & $20.000(15.000,27.625)$ & 194 & $21.000(17.000,29.000)$ & 160 & $26.250(19.000,40.750)$ & $<0.001$ \\
\hline Il-10 & 517 & 164 & $20.000(16.250 ; 25.000)$ & 193 & $20.000(16.000,24.750)$ & 160 & $22.000(17.000,26.000)$ & 0.334 \\
\hline TNFalpha & 520 & 166 & $8.000(7.000,9.000)$ & 193 & $8.000(7.000,9.000)$ & 161 & $8.000(7.000,9.000)$ & 0.444 \\
\hline TGFbeta & 521 & 166 & $8478.75(539,15721.125)$ & 194 & $7623.25(453.5,15302.13)$ & 161 & $7877.0(2252.0,13813.0)$ & 0.664 \\
\hline RENTS & 519 & 165 & $575(396,847.25)$ & 194 & $586.5(446.250,804.750)$ & 160 & $642.25(474.125,877.00)$ & 0.232 \\
\hline ApoAI & 494 & 159 & $1.153 \mathrm{e} 6(9.857 \mathrm{e} 5,1.33 \mathrm{e} 6)$ & 182 & 1.12e6(9.531e5, $1.288 \mathrm{e} 6)$ & 153 & $1.114 \mathrm{e} 6(9.601 \mathrm{e} 5,1.31 \mathrm{e} 6)$ & 0.272 \\
\hline ApoAII & 504 & 161 & $2.953 \mathrm{e} 5(2.48 \mathrm{e} 5,3.768 \mathrm{e} 5)$ & 188 & $2.92 \mathrm{e} 5(2.395 \mathrm{e} 5,3.487 \mathrm{e} 5)$ & 155 & 2.564e5(1.996e5, 3.19e5) & $<0.001$ \\
\hline ApoB & 504 & 163 & $6.490 \mathrm{e} 4(4.750 \mathrm{e} 4,8.32 \mathrm{e} 4)$ & 186 & $5.73 \mathrm{e} 4(3.926 \mathrm{e} 4,8.160 \mathrm{e} 4)$ & 155 & $5.73 \mathrm{e} 4(3.79 \mathrm{e} 4,7.570 \mathrm{e} 4)$ & 0.039 \\
\hline ApoCII & 507 & 162 & $5.055 \mathrm{e} 4(3.58 \mathrm{e} 4,7.393 \mathrm{e} 4)$ & 192 & $4.435 \mathrm{e} 4(2.970 \mathrm{e} 4,6.92 \mathrm{e} 4)$ & 153 & $3.45 \mathrm{e} 4(2.584 \mathrm{e} 4,5.508 \mathrm{e} 4)$ & $<0.001$ \\
\hline ApoCIII & 508 & 163 & $1.66 \mathrm{e} 5(1.128 \mathrm{e} 5,2.392 \mathrm{e} 5)$ & 190 & $1.574 \mathrm{e} 5(1.028 \mathrm{e} 4,2.27 \mathrm{e} 5)$ & 155 & $1.32 \mathrm{e} 5(9.12 \mathrm{e} 4,1.926 \mathrm{e} 5)$ & 0.002 \\
\hline ApoE & 525 & 168 & $51.79(38.962,73.024)$ & 196 & $56.214(40.807,76.496)$ & 161 & $56.696(42.598,80.414)$ & 0.196 \\
\hline ApoJ & 525 & 168 & $140925(2568.2,202690)$ & 196 & $163699(2560.30,206595)$ & 161 & 159364(107898, 188486) & 0.363 \\
\hline ApoH & 526 & 168 & $362980(302895,434305)$ & 196 & $393830(328020,464085)$ & 162 & $386970(343345,453390)$ & 0.008 \\
\hline $\mathrm{TCH}^{\mathrm{a}}$ & 548 & 176 & $4.783 \pm 0.062$ & 203 & $4.574 \pm 0.064$ & 169 & $4.643 \pm 0.071$ & 0.071 \\
\hline TG & 548 & 176 & $1.400(1.000,1.900)$ & 203 & $1.500(1.100,1.900)$ & 169 & $1.300(1.000,1.750)$ & 0.093 \\
\hline HDL-C & 548 & 176 & $0.970(0.830,1.158)$ & 203 & $0.960(0.840,1.110)$ & 169 & $1.050(0.900,1.315)$ & $<0.001$ \\
\hline LDL-C & 548 & 176 & $2.990(2.513,3.518)$ & 203 & $2.740(2.270,3.320)$ & 169 & $2.750(2.145,3.265)$ & 0.004 \\
\hline BMI & 547 & 176 & $24.300(22.800,26.300)$ & 202 & $24.500(22.500,26.925)$ & 169 & $23.500(21.600,26.600)$ & 0.089 \\
\hline Number comorbidity & 547 & 176 & $6.000(4.250,7.000)$ & 202 & $7.000(6.000,8.000)$ & 169 & $7.000(6.000,8.000)$ & $<0.001$ \\
\hline
\end{tabular}

a. For Gaussian distribution continuous data are expressed as mean \pm SEM, ANOVA was used; $b$. For non-Gaussian distribution continuous data are expressed as medians with $25 \%$ and $75 \%$ values in interquartile range. Kruskal-Wallis test was used.

\section{Discussion}

The present study demonstrates that age-dependent alterations in the number of co-morbidities, grade of fundus AS and the profile of systemic inflammation, inflammatory cytokines, oxidative products and antioxidative enzyme activities is a dynamic process. Compared with middle-aged patients ( $\leq 74$ years old), long-living patients ( $\geq 85$ years old) had high risks for a great number of co-morbidities and a higher grade of fundus AS. However, the oldest old patients had favorable lipid profiles, indicated by significantly high serum levels of HDL-C. Moreover, two populations had similar anti-oxidative enzyme activities, systemic inflammatory profiles and inflammatory cytokine levels (Table 3). Compared with older patients (75- to 84 -yearold), the oldest old patients had similar risks for the number of co-morbidities and the grade of fundus AS. However, the oldest old patients had significantly high serum levels of HDL-C and anti-oxidative enzyme activities. Meanwhile, the oldest old patients also had a significantly high risk for systemic inflammation (Table 3).

Our results confirm that the oldest old individuals have high anti-oxidative enzyme activities and high serum levels of HDL-C after adjusting for other parameters. The high serum anti-oxidative enzyme activities observed in the oldest old individuals were in agreement with the results of previous studies (2). In the elderly, the imbalance between pro-oxidants and enzymatic anti-oxidant systems due to antioxidant deficiencies increases their susceptibility to oxidative damage, which accelerates aging and age-related diseases. Genetic polymorphisms in the pro-/antioxidant pathway can influence physical and cognitive performance and the survival of nonagenarians (22). Dietary restriction and redox-based therapeutic intervention to reduce mitochondrial reactive oxygen species production and the global network 
Table 3

Results from multivariate regression analyses (forward stepwise) predicting age-related alteration (adjusting for single categorical variables, sex, ApoE genotype, metabolic syndrome; single continuous variables BMI, LPO other anti-oxidant enzymes, inflammatory cytokines, lipoproteins apolipoproteins)

\begin{tabular}{|c|c|c|c|c|}
\hline & \multicolumn{2}{|c|}{$\leq 74$ years old ${ }^{a}$} & \multicolumn{2}{|c|}{ 75- to 84-year-olda } \\
\hline & OR (95\% CI) P-Value & Global P-Value & OR (95\% CI) P-Value & Global P-Value \\
\hline GSHpx & $1.002(0.985,1.019)$ & 0.813 & $0.986(0.972,1.000)$ & 0.043 \\
\hline Hs CRP I & $4.005(1.019,15.736)$ & 0.047 & $8.516(1.630,44.484)$ & 0.011 \\
\hline Hs CRP II & $1.654(0.441,6.200)$ & 0.456 & $7.699(1.544,38.388)$ & 0.013 \\
\hline Hs CRP III & $1.300(0.295,5.724)$ & 0.729 & $7.251(1.346,39.070)$ & 0.021 \\
\hline Hs CRP IV & $0^{\mathrm{b}}$ & - & $0^{b}$ & - \\
\hline HDL-C & $0.127(0.037,0.433)$ & 0.001 & $0.158(0.054,0.457)$ & 0.001 \\
\hline $\begin{array}{l}\text { Number of co-morbi- } \\
\text { dity }\end{array}$ & $0.757(0.636,0.902)$ & 0.002 & $0.939(0.802,1.099)$ & 0.434 \\
\hline AS level 0 & $26.059(4.705,144.324)$ & $<0.001$ & $1.472(0.221,9.787)$ & 0.689 \\
\hline AS level I & $8.539(3.555,20.513)$ & $<0.001$ & $2.232(1.226,4.062)$ & 0.009 \\
\hline AS level II & $0^{\mathrm{b}}$ & - & $0^{\mathrm{b}}$ & - \\
\hline
\end{tabular}

a. Reference class: $\geq 85$ years old; b.Parameter redundancy and setting is 0 .

of oxidative stress can counteract the progression of aging and extend the life span of animals $(23,24)$. A recent German population-based cohort study showed that reactive oxygen species concentration and redox control status were associated with all-cause mortality adjusted for age, sex, education, smoking, physical activity and alcohol consumption (hazard ratios and 95\% confidence intervals: $1.63[1.01 ; 2.63]$ and 0.68 [0.53; 0.87], respectively). The researchers supposed that systemic inflammation and higher co-morbidity could be intermediate states on the pathway from high reactive oxygen species concentration to mortality (25). A high serum level of HDL-C was a characteristic feature of longevity and was associated with better survival in frail, community-living elderly $(9,13)$. A progressive decline in plasma HDL was associated with elevated CRP and IL-6 levels (10). The high plasma levels of HDL-C in the oldest old individuals in our study were also consistent with the results of previous studies $(9,13)$.

We also demonstrated that the oldest old individuals have high risks for a greater number of co-morbidities, higher grade of fundus AS and systemic inflammation, after adjusting for other parameters. Because the number of abnormal systems has been shown previously to be more predictive of pathological aging than abnormalities in any particular system (26), our study included 18 chronic diseases in more than eight systems. Thus, the greater the number of co-morbidities could reflect a greater degree of aging. Fundus microcirculation changes associated with hypertension, atherosclerosis and increased risk of stroke and myocardial infarction (27). Systemic inflammation was associated with normal aging and coronary atherosclerosis (5). Oxidative stress and chronic inflammation were positively correlated with the grade of sclerotic change in the fundus oculi, promoting atherosclerosis in the retinal arteries (28). In our study, the risk of metabolic syndrome increased with age, but metabolic syndrome was not significantly association with age after adjusting for other factors (Table 1). Chronic subclinical inflammation was associated with poorer physical function in older adults with various co-morbidities (29). Therefore, the oldest old patients showed an increase in age-associated diseases and adverse clinical prognoses.

ApoE genotype, plasma levels of inflammatory cytokines and apolipoprotein, may also play important roles in aging. Our results showed that the older patients had a higher level of inflammatory cytokine IL-6, lower levels of ApoAII, B, CII and CIII, and higher levels of ApoH and LDL-C (Table 2). However, after adjusting for other factors, these age-related differences were not significant. Although the ApoE polymorphism is involved in lipid metabolism regulation, and ApoE \&4 allele frequencies were high in patients with several age-related illnesses $(10,14)$, ApoE $\varepsilon 4$ allele frequencies were low in the the oldest old population (11). Our study showed that there was no significant difference in the age-related distribution of ApoE alleles.

The study provides valuable information to develop biomarkers of aging. The missing values of the study parameters accounted for less than $12 \%$ of the total study subjects. The main limitation of our study was its relatively small sample size, and particularly the low number of females, which weakens the validity of our results. The ruaral citizens is scarce in our sample was another limitation. Furthermore, as this was a cross- 
sectional study, the associations of adverse prognoses, such as disability and death, with changes in the study parameters could not be determined.

In conclusion, the oldest old patients had significantly high anti-oxidative enzyme activities and serum HDL-C levels. Meanwhile, these patients also had significantly high numbers of co-morbidities, high grades of fundus AS and high systemic inflammation, which might predict the degree of aging.

\section{Conflicts of Interest: None declared.}

Funding: This work was supported by grants from the Shanghai Hospital Development Center (No. SHDC12014221).

Ethical standards: The procedures followed in the manuscript were in accordance with the ethical standards of the responsible committee on human experimentation (institutional and national ) and with the Helsinki Declaration of 1975 , as revised in 2000

\section{References}

1. Belenguer Varea A, Mohamed Abdelaziz K, Avellana Zaragoza JA, et al Oxidative stress and longevity; a case-control study. Rev Esp Geriatr Gerontol 2014;pii: S0211-139X(14)00126-127.

2. Mecocci $P$, Polidori MC, Troiano L, et al. Plasma antioxidants and longevity: a study on healthy centenarians. Free Radic Biol Med 2000;28(8):1243-1248.

3. Singh $T$, Newman AB. Inflammatory markers in population studies of aging. Ageing Res Rev 2011;10(3):319-329.

4. Nash SD, Cruickshanks KJ, Klein R, et al. Long-term variability of inflammatory markers and associated factors in a population-based cohort. J Am Geriatr Soc 2013;61(8):1269-1276.

5. Quaglia LA, Freitas W, Soares AA, et al. C-reactive protein is independently associated with coronary atherosclerosis burden among octogenarians. Aging Clin Exp Res 2014;26(1):19-23.

6. Choi WS, Lee WK, Lee SH, et al. Is high-sensitivity C-reactive protein associated with lower urinary tract symptoms in aging men? Results from the hallym aging study. Korean J Urol 2012;53(5):335-41.

7. Labonté ME, Dewailly E, Chateau-Degat ML, et al. Population-based study of high plasma C-reactive protein concentrations among the Inuit of Nunavik. Int J Circumpolar Health 2014;71.

8. Atzmon G, Gabriely I, Greiner W, et al. Plasma HDL levels highly correlate with cognitive function in exceptional longevity. J Gerontol A Biol Sci Med Sci 2002;57(11):M712-715

9. Landi F, Russo A, Pahor M, et al. Serum high-density lipoprotein cholesterol levels and mortality in frail, community-living elderly. Gerontology 2008;54:7178 .

10. Arai Y, Hirose N, Nakazawa S, et al. Lipoprotein metabolism in Japanese centenarians: effects of apolipoprotein E polymorphism and nutritional status. J Am Geriatr Soc 2001;49(11):1434-1441.
11. Garatachea N, Marín PJ, Santos-Lozano A, et al. The ApoE gene is related with exceptional longevity: a systematic review and meta-analysis. Rejuvenation Res 2014 Nov 10. [Epub ahead of print].

12. Bonomini F, Filippini F, Hayek T, et al. Apolipoprotein E and its role in aging and survival. Exp Gerontol 2010;45(2):149-157.

13. Atzmon G, Rincon M, Schechter CB, et al. Lipoprotein genotype and conserved pathway for exceptional longevity in humans. PLoS Biol 2006; 4(4):e113.

14. Motta M, Bennati E, Cardillo E, et al. The significance of apolipoprotein-B (Apo-B) in the elderly as a predictive factor of cardio-cerebrovascular complications. Arch Gerontol Geriatr 2009; 49(1):162-164.

15. Luoma PV. Gene activation, apolipoprotein A-I/high density lipoprotein, atherosclerosis prevention and longevity. Pharmacol Toxicol 1997;81(2):57-64.

16. Lee YN, Shim YJ, Kang BH, et al. Over-expression of human clusterin increase stress resistance and extends lifespan in Drosophila melanogaster. Biochem Biophys Res Commun 2012;420(4):851-856.

17. Trougakos IP, Petropoulou C, Franceschi C, et al. Reduced expression levels of the senescence biomarker clusterin/apolipoprotein $\mathrm{j}$ in lymphocytes from healthy centenarians. Ann N Y Acad Sci 2006;1067:294-300.

18. Ma yongxing, Ruan Qingwei, Zhou Yue, et al. Correlation between cognitive function and ApoE genotypes in the elderly with four different cognitive states. Chinese Journal of Geriatrics 2013,32(08)817-820.

19. Chenillot O, Henny J, Steinmetz J, et al. High sensitivity C-reactive protein biological variations and reference limits. Clin Chem Lab Med 2000; 38(10):1003-1011.

20. Eshtiaghi R, Keihani S, Hosseinpanah F, et al. Natural course of metabolically healthy abdominal obese adults after 10 years of follow-up: the Tehran Lipid and Glucose Study. Int J Obes (Lond). 2014 Oct 7.

21. Kim SW, Heo JH, Kim CH, et al. Rapid and direct detection of apolipoprotein $\mathrm{E}$ genotypes using whole blood from humans. J Toxicol Environ Health A 2010; 73(21-22):1502-1510.

22. Dato S, Soerensen M, Lagani V, et al. Contribution of genetic polymorphisms on functional status at very old age: a gene-based analysis of 38 genes (311 SNPs) in the oxidative stress pathway. Exp Gerontol 2014;52:23-29.

23. Canistro D, Boccia C, Falconi R, et al. Redox-Based Flagging of the Global Network of Oxidative Stress Greatly Promotes Longevity. J Gerontol A Biol Sci Med Sci 2014;pii: glu160.

24. Pincemail J, Ricour C, Defraigne JO, et al. Oxidative stress, antioxydants and the ageing process. Rev Med Liege 2014;69(5-6):270-275.

25. Schöttker B, Saum KU, Jansen EH, et al. Oxidative Stress Markers and AllCause Mortality at Older Age: A Population-Based Cohort Study. J Gerontol A Biol Sci Med Sci 2014;pii: glu111.

26. Fried LP, Xue QL, Cappola AR, et al. Nonlinear multisystem physiologica dysregulation associated with frailty in older women: implications for etiology and treatment. J Gerontol A Biol Sci Med Sci 2009; 64: 1049-1057.

27. De Boever P, Louwies T, Provost E, et al. Fundus Photography as a Convenien Tool to Study Microvascular Responses to Cardiovascular Disease Risk Factors in Epidemiological Studies. J Vis Exp 2014;(92). doi: 10.3791/51904.

28. Sakane N, Fujiwara S, Sano Y, et al. Oxidative stress, inflammation, and atherosclerotic changes in retinal arteries in the Japanese population; results from the Mima study. Endocr J 2008;55(3):485-488.

29. Brinkley TE, Leng X, Miller ME, et al. Chronic inflammation is associated with low physical function in older adults across multiple comorbidities. J Gerontol A Biol Sci Med Sci 2009;64(4):455-461. 